\title{
HuluFISH non-denaturing in situ detection of genomic DNA opened by CRISPR-Cas9 Nickase and Exonuclease
}

\author{
Kang Han ${ }^{1}$, Sheng Liư ${ }^{1}$, Yongsheng Cheng ${ }^{1 *}$ \\ ${ }^{*}$ Corresponding author:yongsheng.cheng@pixelbiotech.com \\ 1. PixelBiotech $\mathrm{GmbH}$, Waldhofer str. 104, 69123, Heidelberg, Germany
}

\begin{abstract}
DNA fluorescence in situ hybridization (FISH) has been widely used in diagnosis and genetic research. Traditional Bacterial artificial chromosome (BAC) or oligonucleotide based probe to detect DNA in situ is only effective when the target is relatively large, usually over $150 \mathrm{~Kb}$ DNA fragments. And it involves heat denaturation steps to open the DNA for in situ hybridization. The heat process can affect the fine structure of nuclei. Here we reported a novel method based on Cas9 nickase and exonuclease digestion of double strand DNA and permanently mark the DNA in single strand state for FISH. With this novel design, we detected non-repetitive genomic loci as small as $2 \mathrm{~Kb}$.
\end{abstract}

\section{Introduction}

DNA FISH is a powerful technique in detecting chromosomal structures and has been widely used in study chromatic structure and clinical diagnosis of chromosomal abnormalities. However, the DNA FISH needs the genomic DNA to be opened by heat denaturation and relatively long target region to generate sufficient hybridization signal for detection. The usual BAC (Bacterial Artificial Chromosome) based DNA FISH probe spans dozens of kilobases (kb) or even hundreds of $\mathrm{kb}$ in genomic DNA. For fine chromatic structure study, the heat denaturation and long target region sometimes are prohibitive, especially when the target region is less than $10 \mathrm{~kb}$ like an exogenous gene introduced into the genome in cell and gene therapy.

The CRISPR-Cas9 (Streptococcus pyogenes) system has been extensively explored in studying genome structure both in vivo and in vitro (Chen et al., 2013; Deng et al., 2015; Ishii et al., 2019; Knight et al., 2015; Wang et al., 2021). The CRISPR-Cas9-sgRNA ribonucleoprotein complex can target a specific loci on genomic and label or open genome for hybridization.

Here we are presenting a novel method (CRISPR-ExoFISH) based on the CRISPR-Cas9 system to open the genome loci and digest the specific loci to be single strand DNA (sSDNA) for permanent accessibility for DNA FISH probes (Figure 1). Once the DNA is opened by CRISPR-Cas9 (H840A mutant) on the target strand (TS), a T7 exonuclease starts to digest the non-targeting strand (NTS) of the CRISPR-Cas9 system and leave the targeting strand intact for hybridization. For the following FISH probe hybridization, heat denaturation is not needed and a 
shorter region below $5 \mathrm{~kb}$ is sufficient for the DNA FISH probe to bind and generate specific signals.
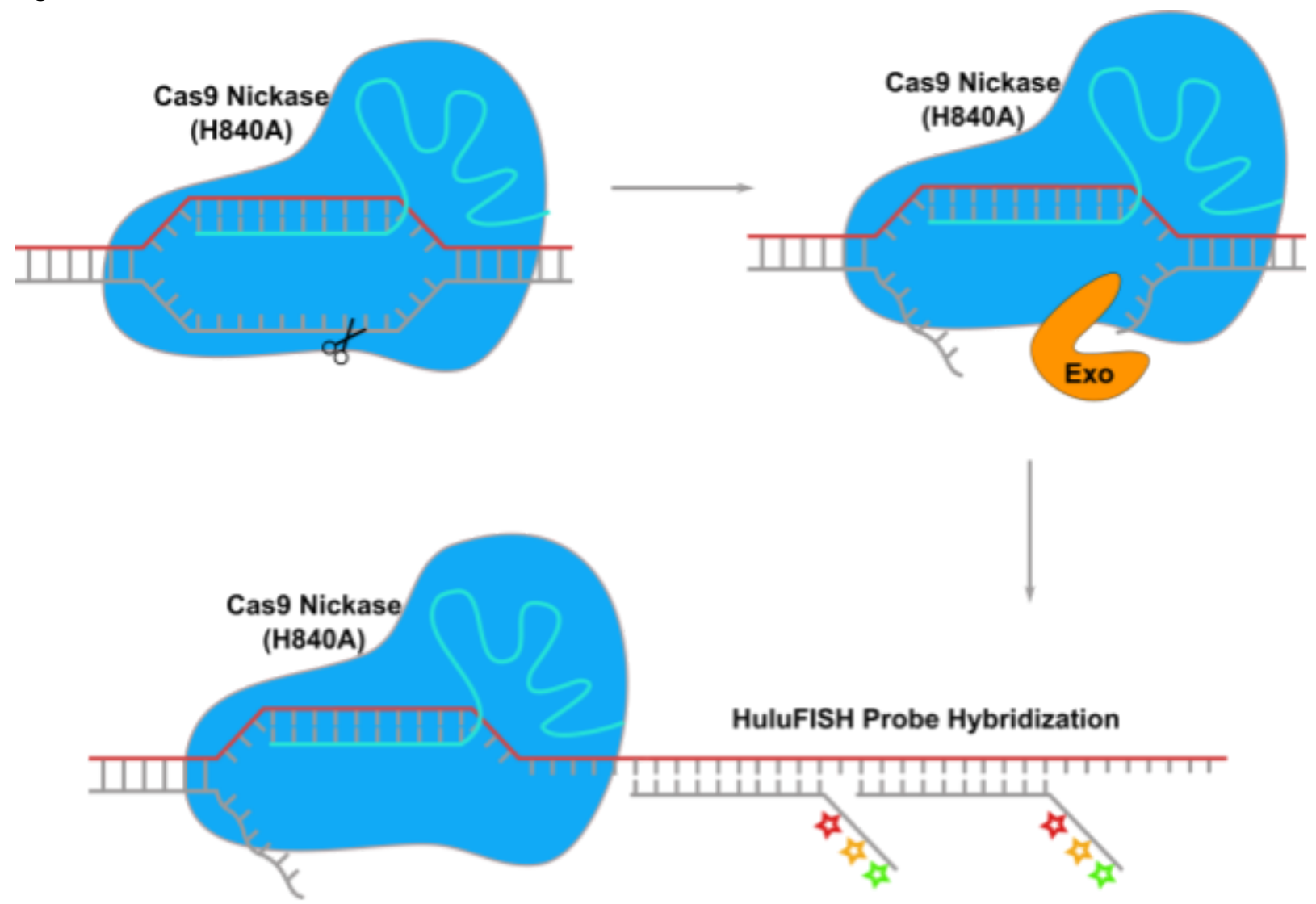

Figure 1. CRISPR-ExoFISH workflow. Genomic DNA loci is binded by Cas9 nickase (H840A)-sgRNA (cyan) ribonucleoprotein complex and cut at NTS strand (grey). Exonuclease find the flapped DNA on NTS strand and digest the NTS stand. Then HuluFISH probe is hybridizing to the TS stand (red).

\section{Method and Materials}

sgRNA synthesis by in vitro transcription (IVT)

sgRNA was produced with the ENGen SgRNA synthesis kit (NEB, E3322). sgRNA guide sequences were designed by customized script at PixelBiotech $\mathrm{GmbH}$ for Human Telomere, alpha-satellite DNA (ASAT), and the non-repetitive region of MUC4 gene (Intron 1, $2 \mathrm{~kb}$ long target). Target-specific DNA oligo templates were designed according to the protocol for ENGen SgRNA synthesis kit. The sgRNA IVT was following the protocol from the kit. For multiple sgRNA synthesis in one reaction, the total concentration of oligo DNA template was adjusted to 250nM final concentration. The sgRNA was purified with the Monarch RNA Cleanup kit (NEB, T2040). 


\section{CRISPR-ExoFISH}

Hela cells were fixed by cold methanol:acetic $\left(3: 1\right.$, stored at $\left.-20^{\circ} \mathrm{C}\right)$ for 10 min and stored at $-20 \mathrm{C}$ or immediately used. RNase A (NEB, T3018) treatment at $0.1 \mathrm{mg} / \mathrm{ml}$ in the $1 \mathrm{xPBS}$ for $30 \mathrm{~min}$ at $37^{\circ} \mathrm{C}$ is optional to remove background staining from cellular RNA. After RNase A treatment, the cells were washed with $1 x P B S$ at $65 \mathrm{C}$ for 10 min each, 3 times. Cas9-sgRNA ribonucleoprotein complex was reconstituted in binding buffer (20mM HEPES, pH 7.5, 100mM KCl, 5mM MgCl2, $5 \%(\mathrm{v} / \mathrm{v})$ Glycerol, $0.1 \%(\mathrm{v} / \mathrm{v})$ Tween-20, $1 \%(\mathrm{w} / \mathrm{v})$ Bovine serum albumin (BSA), $1 \mathrm{mM}$ Dithiothreitol (DTT), 0.4U/ul RNase inhibitor Murine (NEB, M0314)) at the concentration of 200nM each (1:1 ratio for Cas9:sgRNA concentration) for $10 \mathrm{~min}$ at room temperature. Fixed cells were washed with the binding buffer for $10 \mathrm{~min}$ at room temperature. Then the cells were incubated with 200nM Cas9-sgRNA ribonucleoprotein complex in the binding buffer with 1U/ul T7 exonuclease (NEB, M0263) for 1 hour at $37^{\circ} \mathrm{C}$. Then the cells were washed with $1 \times$ PBS for 5 min. After washing with 1 xPBS for 3 times in 5 min, cells were hybridized with a $320 \mathrm{nM}$ HuluFISH probe in HuluHyb (PixelBiotech $\mathrm{GmbH}, \mathrm{PB} 102)$ for 1 hours at $37^{\circ} \mathrm{C}$. After hybridization, the cells were washed with $2 \times S S C, 20 \%$ formamide at $37^{\circ} \mathrm{C}$ for $10 \mathrm{~min}$ for two times, and then washed with 1xPBS for 5 min before mounting in the VectorShield HardSet (Vector Laboratories, $\mathrm{H}-1400)$. Stained cells were imaged under Leica TCS SP8 confocal microscope.

\section{Results}

We tested CRISPR-ExoFISH on 3 targets in human cells. In Figure 2A-B, telomere sgRNA were designed against the repeat TAAGGG in Human telomeres (sequence details in table 1). Without sgRNA and Cas9, HuluFISH probes for telomeres could not bind it all onto the genome without heat denaturation. In the facilitation of Cas9-sgRNA complex, HuluFISH probes efficiently hybridize to the telomere region. This indicates the ssDNA nature of telomeres after Cas9-sgRNA binding and T7 exonuclease digestion. Another repeat region in the genome, alpha satellite DNA (ASAT) was chosen for further validation of this novel method (Figure 2C-D). All repeated genomic regions show specific staining compared to the negative control without Cas9-sgRNA opening and digesting the genomic DNA.

Despite the repeat region, CRISPR-ExoFISH was also employed to detect non-repetitive genomic loci. MUC4 intron 1 was chosen for the validation (Figure 2E-F). A $2 \mathrm{~kb}$ region was selected for sgRNA design and 9 sgRNAs were selected for in vitro transcription and assembly with Cas9. Additionally 63 HuluFISH probes were designed for the $2 \mathrm{~kb}$ region in MUC4. The results showed specific staining of the MUC4 gene intron 1. 

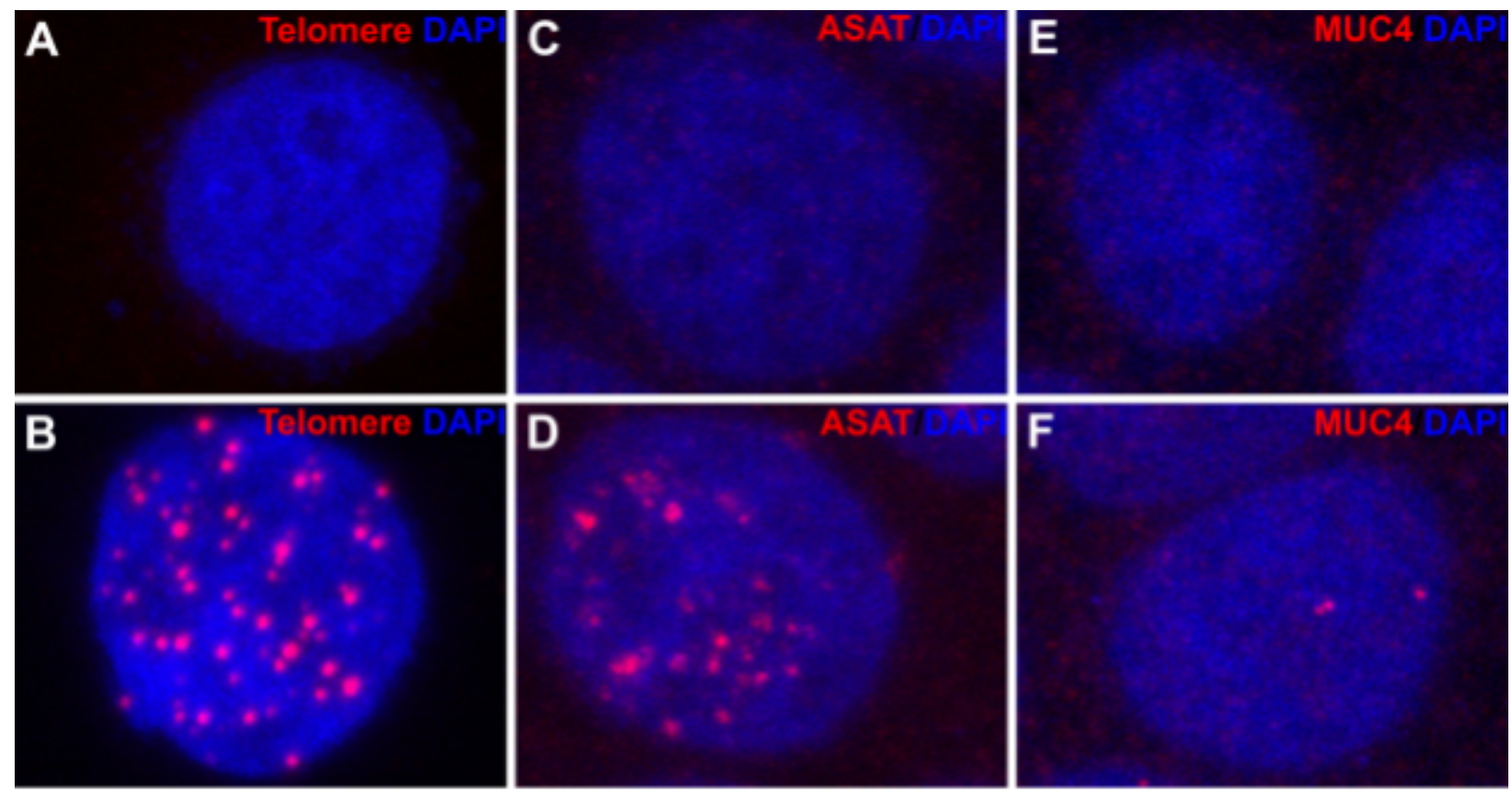

Figure 2. CRISPR-ExoFISH of Human genomic targets. (A, C, E) Hela cell stained with HuluFISH probe for telomere, ASAT, and MUC4 respectively. (B, D, F) Hela cell were fixed and stained with HuluFISH probe against Human telomere, ASAT, and MUC4 respectively, after genomic DNA of telomere, ASAT and MUC4 is opened and digested as ssDNA.

\section{References}

Chen, B., Gilbert, L.A., Cimini, B.A., Schnitzbauer, J., Zhang, W., Li, G.-W., Park, J., Blackburn, E.H., Weissman, J.S., Qi, L.S., et al. (2013). Dynamic Imaging of Genomic Loci in Living Human Cells by an Optimized CRISPR/Cas System. Cell 155, 1479-1491.

Deng, W., Shi, X., Tjian, R., Lionnet, T., and Singer, R.H. (2015). CASFISH:

CRISPR/Cas9-mediated in situ labeling of genomic loci in fixed cells. Proc. Natl. Acad. Sci. 112, $11870-11875$.

Ishii, T., Schubert, V., Khosravi, S., Dreissig, S., Metje-Sprink, J., Sprink, T., Fuchs, J., Meister, A., and Houben, A. (2019). RNA-guided endonuclease - in situ labelling (RGEN-ISL): a fast CRISPR/Cas9-based method to label genomic sequences in various species. New Phytol. 222, 1652-1661.

Knight, S.C., Xie, L., Deng, W., Guglielmi, B., Witkowsky, L.B., Bosanac, L., Zhang, E.T., Beheiry, M.E., Masson, J.-B., Dahan, M., et al. (2015). Dynamics of CRISPR-Cas9 genome interrogation in living cells. Science 350, 823-826.

Wang, Y., Cottle, W.T., Wang, H., Feng, X.A., Mallon, J., Gavrilov, M., Bailey, S., and Ha, T. (2021). Genome oligopaint via local denaturation fluorescence in situ hybridization. Mol. Cell 81, 1566-1577.e8.

Appendix 1 
Table 1 Sequence of oligos used in this study.

Sequence

TTCTAATACGACTCACTATAGGGATGAATAtaagggta Telc agggtaagggtaaGTTTTAGAGCTAGA
Name

Note

TTCTAATACGACTCACTATAGGGATGAATActttttgtag ASAT_gRN Oligo DNA template for aatctgcaagGTTTTAGAGCTAGA

$A$

sgRNA in alpha satellite

TTCTAATACGACTCACTATAGGGATGAATAgcgatgtc MUC4NR_ Oligo DNA template for tggaaggatccaGTTTTAGAGCTAGA gRNA_7 sgRNA in MUC4 intron 1

TTCTAATACGACTCACTATAGGGATGAATAggtgaga cgcagacaaactgGTTTTAGAGCTAGA

TTCTAATACGACTCACTATAGGGATGAATAttgggtca cggccttgcctgGTTTTAGAGCTAGA

TTCTAATACGACTCACTATAGGGATGAATActggggtc MUC4NR_ Oligo DNA template for cagagttcaagcGTTTTAGAGCTAGA

gRNA_620 sgRNA in MUC4 intron 1

TTCTAATACGACTCACTATAGGGATGAATAcaggact aggctgagggcaaGTTTTAGAGCTAGA

MUC4NR_ Oligo DNA template for gRNA_821 sgRNA in MUC4 intron 1

MUC4NR

TTCTAATACGACTCACTATAGGGATGAATAgaggctg gRNA_10 gggcttggggcgcGTTTTAGAGCTAGA 5

Oligo DNA template for sgRNA in MUC4 intron 1

MUC4NR

TTCTAATACGACTCACTATAGGGATGAATAaagctga gRNA_12 gcccaaatgtgctGTTTTAGAGCTAGA 5 Oligo DNA template for sgRNA in MUC4 intron 1

MUC4NR

TTCTAATACGACTCACTATAGGGATGAATActgagtcctgRNA_14 ttgcgtcgctaGTTTTAGAGCTAGA

4

Oligo DNA template for

MUC4NR

TTCTAATACGACTCACTATAGGGATGAATAacctgcct gRNA_16 $\overline{6}$ atacttgagtctGTTTTAGAGCTAGA

2 sgRNA in MUC4 intron 1

MUC4NR

TTCTAATACGACTCACTATAGGGATGAATAgatggaa gRNA_187 attggtgtctacGTTTTAGAGCTAGA

0

Oligo DNA template for sgRNA in MUC4 intron 1

HuluFISH_HuluFISH probe sequence Telomere for Telomere

ASAT_GSOHuluFISH probe sequence

CTCACAGAGTTGAACCTTCCT

_AS for alpha satellite DNA

CCCAGGCTTACTCGCAGA
MUC4_NR HuluFISH probe MUC4 intron 
GAGAAAGACAGGCAGAGC

CAGAGCAAGAGGAACAGAGTC

AAGGAGAAAGATGTACACCC

TTGTGTACAGAGCTGGGGG

TAGAGGGGATGCCAGGAAAG

CTGGGTGATGGAGACGGAAG

AACTCATGTAAAGCTGCAGGG

AGGACCCTGGGAATGGAGA

GAGGAGAAGATCGGGAG

CAGCAAGCAAGGGAAGC

ACAAGGAGGAGAGGGGCA

CCGTGGGCTTCTTACCTCTG

AGCTCGGGTTTAAAAGC

CCTTGGGCTCCTCCGTGTACA

CCAGGCCTGTGGGAGATGTT

CCCGTCCTCTTCCCCACACTT

GCTGTCCCTCTGGCTTCA

ATTCTGTGTTTCTGGGTGTC
-52 1

MUC4_NR HuluFISH probe MUC4 intron -70 1

MUC4_NR HuluFISH probe MUC4 intron -91 1

MUC4_NR HuluFISH probe MUC4 intron -111 1

MUC4_NR HuluFISH probe MUC4 intron $\begin{array}{ll}130 & 1\end{array}$

MUC4_NR HuluFISH probe MUC4 intron _150 1

MUC4_NR HuluFISH probe MUC4 intron -170 1

MUC4_NR HuluFISH probe MUC4 intron -193 1

MUC4_NR HuluFISH probe MUC4 intron -247

MUC4_NR HuluFISH probe MUC4 intron -264 1

MUC4_NR HuluFISH probe MUC4 intron _285 1

MUC4_NR HuluFISH probe MUC4 intron _304 1

MUC4_NR HuluFISH probe MUC4 intron _385 1

MUC4_NR HuluFISH probe MUC4 intron -402 1

MUC4_NR HuluFISH probe MUC4 intron

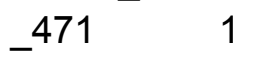

MUC4_NR HuluFISH probe MUC4 intron _511 1

MUC4_NR HuluFISH probe MUC4 intron _544 1

MUC4_NR HuluFISH probe MUC4 intron _567 1

MUC4_NR HuluFISH probe MUC4 intron 
TGAGTCTTTGGGGGAGAGT

TGGGGTTAGAGTCTCAGCTCC

CCTCTCAGCAGGCTAAGAACA

GTCGCCGAGGGAAAATATTTC

TTGGGCGCATATTTGAGGAG

CTTCCTGGGAGTGAGTCAGA

CGAGTGCCGTTTAAAGG

CTGCAAGAGAAGCCATGCTG

CCTTCCACCTCGGGATGTT

GCTCCAGTGCTCATCCCA

TGCCCTGCAGATGTGGTTG

GGAGTCTCCACGGGAATCA

CGGGGAGGCAGCTTGGATTT

CCCCAGGGCCAAGTCTTT

GCCGTGAACTGTTCTGG

GCCCCGGTCCCCTGTGTAAAA

AGCAGTGGTGAACGGTTGG

ACCTCCTGACGCCCAAGTT
599
-1

MUC4_NR HuluFISH probe MUC4 intron -618 1

MUC4_NR HuluFISH probe MUC4 intron -659 1

MUC4_NR HuluFISH probe MUC4 intron -687

MUC4_NR HuluFISH probe MUC4 intron _708 1

MUC4_NR HuluFISH probe MUC4 intron -728 1

MUC4_NR HuluFISH probe MUC4 intron -748 1

MUC4_NR HuluFISH probe MUC4 intron _768 1

MUC4_NR HuluFISH probe MUC4 intron -788

MUC4_NR HuluFISH probe MUC4 intron _818 1

MUC4_NR HuluFISH probe MUC4 intron _929 1

MUC4_NR HuluFISH probe MUC4 intron -989

MUC4_NR HuluFISH probe MUC4 intron ${ }_{-} 1010 \quad 1$

MUC4_NR HuluFISH probe MUC4 intron -1063 1

MUC4_NR HuluFISH probe MUC4 intron -1086 1

MUC4_NR HuluFISH probe MUC4 intron _1103 1

MUC4_NR HuluFISH probe MUC4 intron $1160 \quad 1$
-

MUC4_NR HuluFISH probe MUC4 intron -1179 1

MUC4_NR HuluFISH probe MUC4 intron 
CTGGACACTCAGCTCCATGT

CAGGAAGTGGGGTCTGTGGA

ACCTGGGGTGGGCGTGGAAAA

CGTGACCCGGAGAAGGAGTGA

AGGACTGTTGGTGTGCAAGG

GTCTCCATGACGACCCGAA

GAAGCTAGGCATGTCGTGGA

AAGTGGAGCTGGGCCAGGAGA

GAGATGGTCGTGGCTGGGAGA

TGGCACCCACACATCTGAC

CTTGGCAGGAAAAGCAGTCAC

CCAGCCAGCCCCGTTCCTTTT

CCTGAAGGGGTTTACAGATG

AGGGTCTGTTTGCACACTTG

CCAGGCTGGGTCACACTGAA

GCCCAGGCCAGAGGAAAAA

CACAGGGTTTCCACAAAG

GCTGCCGCAATGAGGGTTT
1198
1

MUC4_NR HuluFISH probe MUC4 intron $-1272 \quad 1$

MUC4_NR HuluFISH probe MUC4 intron _ $1297 \quad 1$

MUC4_NR HuluFISH probe MUC4 intron _1338 1

MUC4_NR HuluFISH probe MUC4 intron ${ }_{-1368} 1$

MUC4_NR HuluFISH probe MUC4 intron -1388 1

MUC4_NR HuluFISH probe MUC4 intron -1409 1

MUC4_NR HuluFISH probe MUC4 intron $\begin{array}{ll}-1429 & 1\end{array}$

MUC4_NR HuluFISH probe MUC4 intron $\begin{array}{ll}1481 & 1\end{array}$

MUC4_NR HuluFISH probe MUC4 intron -1503 1

MUC4_NR HuluFISH probe MUC4 intron $\begin{array}{ll}1522 & 1\end{array}$

MUC4_NR HuluFISH probe MUC4 intron _1559 1

MUC4_NR HuluFISH probe MUC4 intron -1635 1

MUC4_NR HuluFISH probe MUC4 intron _1660 1

MUC4_NR HuluFISH probe MUC4 intron _1700 1

MUC4_NR HuluFISH probe MUC4 intron $-1736 \quad 1$
-10404

MUC4_NR HuluFISH probe MUC4 intron _1755 1

MUC4_NR HuluFISH probe MUC4 intron ${ }_{-1773} 1$

MUC4_NR HuluFISH probe MUC4 intron 
CCTTAAGGAACAGCCCTGGC

CAAGGGTTAAAGGATAAGGC

CAGCAGACAGAGGTGGGCTA

CAGGCAGGAATGACTCAGA

CCGTCCTGGTTCCATGCCACA

GGCAACTGGGTCGAAATAGG

CCTTGGTCTCCAGCACTATCA
$1794 \quad 1$
-

MUC4_NR HuluFISH probe MUC4 intron -1814 1

MUC4_NR HuluFISH probe MUC4 intron -1837 1

MUC4_NR HuluFISH probe MUC4 intron -1858

MUC4_NR HuluFISH probe MUC4 intron -1916 1

MUC4_NR HuluFISH probe MUC4 intron -1948 1

MUC4_NR HuluFISH probe MUC4 intron -1974 1

MUC4_NR HuluFISH probe MUC4 intron _ $1995 \quad 1$ 